\title{
Prognostic role of troponin and natriuretic peptides as biomarkers for deterioration of left ventricular ejection fraction after chemotherapy
}

\author{
Paweł Stachowiak ${ }^{1}$, Zdzisława Kornacewicz-Jach ${ }^{1}$, Krzysztof Safranow ${ }^{2}$
}

1Department of Cardiology, Pomeranian Medical University, Szczecin, Poland 2Department of Biochemistry and Medical Chemistry, Pomeranian Medical University, Szczecin, Poland

Submitted: 4 September 2012

Accepted: 22 December 2012

Arch Med Sci

DOI: 10.5114/aoms.2013.34987

Copyright $\odot 2013$ Termedia \& Banach

\author{
Corresponding author: \\ Dr Paweł Stachowiak \\ Department of Cardiology \\ Pomeranian Medical University \\ 72 al. Powstańców Wlkp. \\ 70-111 Szczecin, Poland \\ Phone: +48914661378 \\ Fax: +48 914661379 \\ E-mail: \\ stachowiak22@gmail.com
}

\begin{abstract}
Cardiotoxicity due to anthracyclines, trastuzumab and other potential cardiotoxic drugs is still a problem of modern chemotherapy. For years researchers have tried to find biological markers that can predict changes in the heart. The most thoroughly tested markers are troponin and natriuretic peptides. Some studies have proven that these markers can indeed be useful. In studies which have shown the predictive role of troponin I the assessment of this marker was performed very frequently. It is not possible to carry out such serial measurements in many centers because of typical 1-day hospital stay times. The predictive role of natriuretic peptides still needs further investigation. This review considers the newest research from recent years.
\end{abstract}

Key words: biological markers, cardiotoxicity, chemotherapy, left ventricular dysfunction.

\section{Introduction}

The effectiveness of oncological treatment increases with each decade. However, the efficacy is undermined by potentially life-threatening cardiotoxicity. Cardiotoxicity includes a wide range of cardiac effects from small changes in blood pressure and arrhythmias to cardiomyopathy. Its incidence varies and is subject to different clinical definitions; however, some researchers report the incidence to be as high as 65\% [1]. There are many drugs that can cause impairment of the heart function. Anthracycline antibiotics are potent anti-tumor agents used in a wide spectrum of malignancies. They are part of the gold standard adjuvant therapy for breast cancer and in metastatic disease. They provide significant increases in response rate, time to disease progression, and overall survival. The successful use of anthracyclines is, however, restricted by the risk of developing life-threatening congestive heart failure (HF) [2]. Other cytotoxic drugs that have reported cardiotoxicity include 5 -fluorouracil, capecitabine, mitoxantrone, cisplatin, the taxoids paclitaxel and docetaxel, and newer drugs such as the monoclonal antibody trastuzumab [3-7].

Three distinct types of anthracycline-induced cardiotoxicity (i.e. acute, subacute and chronic) have been described [8]. Acute or subacute effects, such as acute failure of the left ventricle, can occur immediately after treat- 
ment. Also the development of heart failure many years after the last administration of chemotherapeutic drugs is increasingly recognized $[9,10]$. Acute, subacute and chronic cardiotoxicity may lead to development of overt, refractory heart failure with a very bad prognosis [11].

The mechanism by which anthracyclines induce cardiomyopathy is believed to involve the generation of reactive oxygen species, in part through an iron-dependent pathway. The heart is especially vulnerable to this reactive oxygen species generation because cardiac muscle has relatively low levels of antioxidant enzymes [12].

The mechanism of troponin and natriuretic peptides release after chemotherapy needs further definition. Several mechanisms, particularly related to mitochondria, are proposed as being responsible for the increased sensitivity of the heart to doxorubicin-induced toxicity, including:

- higher mitochondrial density per unit volume in cardiomyocytes when compared to other tissues,

- the elevated affinity of doxorubicin to cardiolipin, a major phospholipid component of the inner mitochondrial membrane in the heart,

- the possible existence of a specific, although controversial, NADH dehydrogenase in the heart, which also contributes to anthracycline redox cycling and increased formation of reactive oxygen species [13].

In a mouse model impaired cardiac function was accompanied by the up-regulation of endothelin-1 expression at the mRNA and protein level. Moreover, bosentan, an endothelin receptor antagonist, applied in a pretreatment procedure in mice, strikingly inhibited doxorubicin-induced cardiotoxicity with preserved indices of contractility [14]. That can suggest a role of endothelin receptor in cardiotoxicity.

Liposomal doxorubicin has also been reported as having some protective role in decreased incidence of heart failure. Liposomal anthracyclines have the potential for more selective uptake by cancer cells and reduced cardiac toxicity [15]. A positive role in heart failure protection has also been reported in dexrazoxane treatment. Dexrazoxane therapy was associated with a large and statistically significant reduction in the incidence of myocardial injury, as indicated by troponin T elevations, in doxorubicin-treated children with highrisk acute lymphoblastic leukemia [16].

However, despite some cardiac benefits, a number of issues have created uncertainty about the role of dexrazoxane in both adults and children, including the possibility of a lower anti-tumor response rate [17]. Another trial conducted in women treated with doxorubicin or epirubicin for breast cancer reported the possibility that dexrazoxane may interfere with cancer therapy [18].
Guidelines of the American Society of Clinical Oncology do not recommend dexrazoxane for adults who will receive anthracyclines in an adjuvant therapy, because of concerns about the potential impact on antitumor efficacy [19]. In pediatric malignances this drug is not recommended either. It can be considered in patients with metastatic breast cancer and other malignancies, who have received more than $300 \mathrm{mg} / \mathrm{m}^{2}$ doxorubicin and who may benefit from continued doxorubicin-containing therapy [19].

The standard reference method for monitoring drug-induced cardiac toxicity is the measurement of left ventricular ejection fraction (LVEF). This implies serial LVEF measurements, which are expensive. Decline of the LVEF often leads to further cardiac function impairment. Some studies have shown that elevation of biological markers can precede left ventricular dysfunction [20-22]. Therefore, biological monitoring by serial assays might be a useful method of selecting those patients who require LVEF measurements [23]. For almost the last two decades scientists have been attempting to find a biochemical prognostic marker of imminent heart dysfunction. There has been increased interest in the potential use of biomarkers such as troponin I and natriuretic peptides as predictors of early myocardial damage and incipient heart failure [24]. Troponin T and I are components of the troponin complex of muscle cells used as markers of myocardial damage in suspected myocardial infarction (MI) [25]. Natriuretic peptides, especially natriuretic peptide type $B$ (BNP), is released chiefly by the cardiac ventricles in response to myocardial stresses and correlates well with impairment of systolic and diastolic cardiac function in heart failure [26-29].

There have been many studies about the role of biochemical markers in cardiotoxic chemotherapy. However, various differences between these investigations concerning e.g. the type of troponin, diagnostic cut-off values or drugs used in chemotherapy schemes have been reported. This review describes the latest research into biochemical markers and their predictive role in subsequent cardiac function deterioration. An electronic search of the MEDLINE and PubMed databases (January 1990 to January 2012) was performed to identify studies which analyzed biological markers such as troponin and natriuretic peptides and their predictive role in left ventricular function deterioration.

\section{Troponin - is it really a good prognostic marker of cardiotoxicity in every patient?}

Troponins are well-known cardiac biomarkers of ischemia [30]. The types of troponin used in cardiology are troponin T and I. Although these contractile proteins are found in all myocytes, the troponin $\mathrm{T}$ and troponin I found in myocardial cells are dis- 
tinct from those found in skeletal muscle [31]. Usually they are used in cardiology for diagnosis of myocardial infarction. The small release of troponins during chemotherapy indicates that only a minimal acute necrosis occurs, as compared to that observed in acute cardiac syndromes [20]. Drugs which are used in chemotherapy can damage cardiac cells, leading to release of troponins into the blood. This phenomenon has recently been described in numerous studies. Nowadays clinical interest has been focusing on the predictive role of these markers for heart failure, which is one of the most life-threatening adverse effects of chemotherapy.

Among the first authors to report release of troponin in animals were Herman et al., who examined the influence of doxorubicin on serum level of cTnT in hypertensive rats. Increases in serum levels of cTnT and myocardial lesions were found in the rats. In their study the average cTnT levels and the cardiomyopathy scores correlated with the cumulative dose of doxorubicin [32]. The cumulative dose of doxorubicin is a well-defined risk factor of cardiotoxicity which was also proven in previous studies [33].

The authors who described the elevation of troponin in humans were Lipshultz and collaborators. Their studies conducted in children with acute lymphoblastic leukemia showed increased levels of troponin T during cancer treatment [16, 21]. In this research the magnitude of troponin elevation predicted left ventricular dilatation and wall thinning 9 months later.

Scientists who played a major role in drawing public attention to the use of troponin as a prognostic marker for LVEF were Cardinale et al. The Italian researchers in a few articles reported a predictive role of troponin I. They examined 1548 patients. In all of their studies in the troponin positive group (cTnl+) there was evidence of a significant reduction in LVEF. In one of the first studies by these authors $29 \%$ of patients with cTnl+ had further LVEF values of less than $50 \%$ [20]. The follow-up duration for this study was 9 months. It is worth noting that in the troponin negative group (cTnl-) there was also a significant reduction of LVEF at 3 months, which was not as great as the reduction in the cTnl+ group. This transient decrease was followed by a recovery to baseline levels at 4 and 7 months [20]. In another study, a significant reduction in LVEF was observed after the first month of follow-up in the cTnl+ group. Thereafter, LVEF further worsened during the follow-up period. In the same study the cTnl-group did not show any significant decrease in LVEF during the entire period of observation [34].

In later research these authors obtained similar outcomes: decrease in LVEF was more evident in the group of cTnl-positive patients up to $-18.2 \%$ after 12 months vs. $-2.5 \%$ in the cTnl-negative group [35]. In the largest study, which included 703 cancer patients, Cardinale et al. focused on the prognostic value of troponin I [36]. In this study the follow-up was up to 42 months after the first drug administration. In this time the authors observed in $16 \%$ of enrolled patients cardiac events such as sudden death $(0.4 \%)$, cardiac death $(0.3 \%)$, acute pulmonary edema (0.4\%), asymptomatic LV dysfunction (5\%), life-threatening arrhythmias (2\%) and conduction disturbances requiring a pacemaker $(0.3 \%)$, and heart failure $(7 \%)$ - which was the most frequent cardiac event reported. In this study $22.6 \%$ of patients had at least $15 \%$ degree of LVEF reduction. Among this group 33.3\% had early (5 successive samples during the 3 days after chemotherapy infusion) or late (1 month after the last drugs administration) troponin-positive results. Most of them (59.1\%) had only early-positive results and barely $7.5 \%$ of patients in this group had troponin-negative results both early and late [36].

Another study was focused on trastuzumabinduced cardiotoxicity (TIC) [22]. In the study, TIC occurred in $17 \%$ of patients, and cardiotoxicity occurred more frequently in patients with lower baseline LVEF, with Tnl+ at baseline or during trastuzumab treatment and with metastatic disease. The TIC incidence was higher in patients previously treated with taxanes and anthracyclines. In this study the previous cumulative dose of anthracyclines in patients who developed TIC was significantly higher in TIC patients and was equal to $241 \mathrm{mg} / \mathrm{m}^{2}$ vs. $210 \mathrm{mg} / \mathrm{m}^{2}$ in the group who exhibited no signs of TIC. Finally, patients treated with trastuzumab alone showed a lower incidence of cardiotoxicity compared with those treated with trastuzumab in combination with other agents [22]. Time of follow-up in this study was up to 79 months.

It is worth noting that in most of Cardinale et al.' studies, patients in CTnl-positive groups had prior anthracycline therapy $[20,22,34,35]$. It can be speculated that, with their patients, the previous treatment with anthracyclines might have played either a synergic or a cumulative role. Therefore it is possible that cTnl elevation could be a consequence of prior subclinical changes that may have happened during prior anthracycline therapy. In one [36] of the studies by these authors in contrast to previous studies, troponin-negative patients had previous anthracycline therapy more frequently than in groups with troponin elevation. The authors suggested that the lower incidence of cTnl positivity among patients previously treated with anthracyclines could be a result of previous treatment with anthracyclines (AC) at a lower dose - unfortunately they did not report the dose [36]. Notably, the cumulative dose of anthracyclines (previous anthra- 
cycline dose plus that included in high-dose chemotherapy - defined as therapy which causes $5 \%$ incidence of cardiotoxicity and is an equivalent of $400 \mathrm{mg} / \mathrm{m}^{2}$ of doxorubicin [37]) was similar in all groups in this particular study $\left(484 \mathrm{mg} / \mathrm{m}^{2}, 492\right.$ $\mathrm{mg} / \mathrm{m}^{2}$ and $499 \mathrm{mg} / \mathrm{m}^{2}$ in Tnl-negative, Tnl-early positive and $\mathrm{Tnl}$-late positive patients respectively) [36]. In all of these studies plasma cTnl concentration was measured before and immediately after, and then $12,24,36$, and $72 \mathrm{~h}$ after every cycle of drug administration. None of the other authors measured troponin level so frequently. This could be a key element increasing the chance to find troponin-positive patients. A serious limitation of this scheme is that nowadays 1-day treatment is mostly performed in many centers. Furthermore, this scheme of troponin assessment can be quite expensive, even compared with standard echocardiography.

In one of the studies Cardinale et al. measured troponin I also 1 month after the last administration of chemotherapy (late troponin). In patients with positive late troponin they found greater LVEF maximal reduction during follow-up [36]. Another study that was carried out by Feola et al. did not confirm this association [38]. The hypothesis that late (1 month) elevation of plasma troponin I might predict cardiac events, such as asymptomatic LV dysfunction or life-threatening arrhythmias, was refuted [38]. The authors in this study enrolled 53 patients with early breast cancer treated with cardiotoxic chemotherapy. As opposed to Cardinale et al.' study none of these patients had previous anthracycline therapy. That study was conducted on patients at older age (median was 55.3 years old vs. 47 in Cardinale et al.' study). Also the median chemotherapy dose of AC was distinctly lower in Feola's study and was equal to $540 \mathrm{mg} / \mathrm{m}^{2}$ of epirubicin, which is the equivalent of $270 \mathrm{mg} / \mathrm{m}^{2}$ of doxorubicin vs. $>480 \mathrm{mg} / \mathrm{m}^{2}$ in the Italian study [39]. This could be a major cause of refuting the hypothesis that late elevation of troponin I might predict cardiac outcome in their patients.

Auner et al. also suggest the predictive role of troponin in chemotherapy. They published their analysis performed on 78 patients with hematological malignancies receiving anthracyclines [40]. In this study elevations of cardiac troponin T were assessed. They made serial measurements of serum cTnT levels. The assessment was done $48 \mathrm{~h}$ after the beginning of the therapy, and one measurement was taken every $48 \mathrm{~h}$ thereafter. Increase in cTnT was observed in $15 \%$ of patients and the median was observed on day +21.5 after initiation of anthracycline dosage. In this study the authors lost 50 patients. In the end there were 28 patients in the follow-up stage, of whom $25 \%$ were cTnT+ and $75 \%$ were cTnT negative. Patients with raised cTnT showed a significantly greater decrease in
LVEF than those without cTnT elevation. A serious drawback of this study was the formula used for measurement of the ejection fraction. The authors used the Teichholz formula, which is not recommended for clinical practice [41]. Therefore the conclusions from this study are disputable.

No other data confirmed that the rise of troponin could precede LVEF decrease. In turn, Kilickap et al. examined 41 patients with diagnosis of solid or hematological malignancy who received cardiotoxic chemotherapy. They used troponin $T$ as a prognostic marker. Assessment of troponin was on the $3^{\text {rd }}$ to $5^{\text {th }}$ days following the first course and after the last course of chemotherapy [42]. cTnT levels measured after completion of therapy were significantly higher compared with those measured at baseline and after the first cycle of therapy. Left ventricular ejection fraction did not change in any patient. The authors observed deterioration of diastolic function after treatment. There was no association between cumulative anthracycline doses and diastolic function impairment. There was a twofold decrease in the E/A ratio in those cases where cTnT levels were increased during therapy, compared with those the cTnT levels of which did not change. Also isovolumetric relaxation time (IRT) was prolonged in all patients who had CTnT levels elevated after therapy [42]. Several studies indicate that diastolic dysfunction precedes reduced left ventricular (LV) ejection fraction or cardiac output [43-45]. Therefore deterioration of LVEF cannot be excluded in a longer study; however, this is only an assumption.

Dodos et al. in their study did not find a predictive role of troponin in LVEF deterioration [46]. In this study cTnT levels did not exceed the upper limit of the normal range in any patient. Only $7 \%$ of all patients had low-level elevation of cTnT. Only 1 of these patients developed a concomitant decrease in LVEF. The authors in this research performed a series of CTnT measurements on the $3^{\text {rd }}$ to $5^{\text {th }}$ day following the first administration of anthracycline and after the last course of chemotherapy at 24 and $72 \mathrm{~h}$, and then after 1, 6 and 12 months.

Over the last 2 years a few articles have been published about troponin during chemotherapy. In one of them the American authors enrolled 95 patients with early breast cancer with overexpressed HER-2. The majority of them had detectable cTnl during the study. The timing of cTnl increase preceded a maximum recorded decline in LVEF. However, maximum cTnl levels did not correlate with LVEF declines [47]. The authors suggest that there were several important factors to consider: the event rate was low (3\% had heart failure) and declines in LVEF were relatively uncommon, thereby limiting the statistical power. Researchers 
had a high ( $46 \%$ of patients) drop-out rate, mainly due to diarrhea, as many patients with toxicity did not continue biomarker assessment and many were noncompliant with blood draws up to 18 months [47].

McArthur et al. studied a group of patients treated with bevacizumab and doxorubicin-cyclophosphamide followed by paclitaxel in early-stage breast cancer. Seven patients (9\%) experienced either a symptomatic LVEF decline or an asymptomatic LVEF decline. Results of this study show that maximum cTnl did not exceed the cut-off in $21 \%$ of patients, was detectable in $71 \%$ and elevated in $8 \%$. There was no association between maximum LVEF change and maximum troponin. The authors note that because they drew samples prior to chemotherapy administration (potentially at nadir time points), they could have missed the maximal impact on CTnl [48].

Finally Goel et al. in their study examined 36 patients with breast cancer receiving trastuzumab. In this study troponin I was not elevated in any patient. However, a limitation of this research was the timing of blood sample collection, which was taken immediately before and after $24 \mathrm{~h}$ of cardiotoxic drug infusion. Also troponin I assay had a lower limit of detection $(0.20 \mathrm{mg} / \mathrm{l})$, which is not sensitive to minor but potentially significant fluctuations [49]. A comparison of studies concerning troponins as prognostic markers for LVEF decrease is provided in Table I.

\section{Natriuretic peptide testing: where is the proper cut-off?}

BNP is a cardiac hormone that is mainly expressed in the heart, where its concentration is considerably higher than in the human or rodent brain [50]. It is well known that mechanical stress, such as pressure and volume overload, neurohumoral factors, and cytokines stimulate the gene expression of BNP and levels of myocardial BNP mRNA. Circulating BNP and N-terminal proBNP (NTproBNP) are remarkably increased in patients with congestive heart failure [51]. The cardiomyocytes synthesize a pre-propeptide (preproBNP with 134 amino acids) which is split into a signal peptide and a propeptide (proBNP with 108 amino acids). During secretion from the cardiomyocytes, proBNP is split at a ratio of $1: 1$ into the physiologically active BNP (32 amino acids) which corresponds to the C-terminal fragment, and the biologically inactive $\mathrm{N}$-terminal fragment (NT-proBNP, 76 amino acids) [52]. Natriuretic peptide levels are closely related to HF severity; they are particularly increased in more advanced New York Heart Association (NYHA) classes and in patients with poor outcome [53]. For clinicians, the diagnostic value for BNP or NT-proBNP is similar; the difference is in the cut-off values which are defined by the manufacturer. Physicians have great expectations for these markers as the future of cardio-oncology.

Many articles have shown the usefulness of natriuretic peptides as early biomarkers of cardiotoxicity due to cancer treatment consisting of cardiotoxic drugs such as anthracyclines and trastuzumab. One of the first authors to focus on the possible use of natriuretic peptide type B (BNP) to assess the cardiac state after anthracycline administration was Suzuki et al. In their study BNP levels during treatment increased. Increases in BNP levels correlated with E/A ratio increases, which may suggest raised BNP level to be indicative of induced diastolic dysfunction [54]. It was found that BNP increased during anthracycline treatment but that the increase was transient and thus not predictive of the clinical course. And only those individuals in whom BNP remained elevated developed overt heart failure, which also suggests a potential of BNP in long-term follow-up, albeit not as a guide for anthracycline interruption [54]. In another study, Nousiainen et al. found no significant correlations between any echo parameters and natriuretic peptides until the cumulative doxorubicin dose became very high and reached $500 \mathrm{mg} / \mathrm{m}^{2}$ [55]. Correlations between cumulative doses and BNP concentrations were also shown in another study conducted by French researchers [23]. It indicates that BNP levels increase when heart cells have already been damaged, which is more probable after high-dose chemotherapy [1, 10, 33, 37].

Meinardi et al. in another study showed that during chemotherapy, concentrations of natriuretic peptides in plasma increase, and LVEF decreases, but they did not focus on the predictive role of natriuretic peptides in deterioration of left ventricular function [56]. Then Daugaard et al. found that neither baseline levels of N-ANP or BNP nor changes in the same variables during therapy were predictive of a change in LVEF [57]. Indeed, BNP increase correlated with decline in LVEF but change in BNP did not precede deterioration of LVEF. So natriuretic peptides were biochemical markers of hemodynamic changes to the heart, the result of which is a decline in $\operatorname{LVEF}[56,57]$.

Also Cil et al. did not find any significant differences in LVEF and NT-proBNP levels between patients who had high NT-proBNP levels and those who had normal NT-proBNP levels before chemotherapy [58]. It is worth noting that their group was quite small; the authors enrolled 33 patients.

Tanindi et al. in their study did not find a predictive role of increasing NT-proBNP but in this research none of the patients had any symptoms of clinical heart failure, though the enrolled group had only 37 patients, besides which, the time of follow-up was also quite short and finished on the $45^{\text {th }}$ day after the beginning of therapy [59]. 


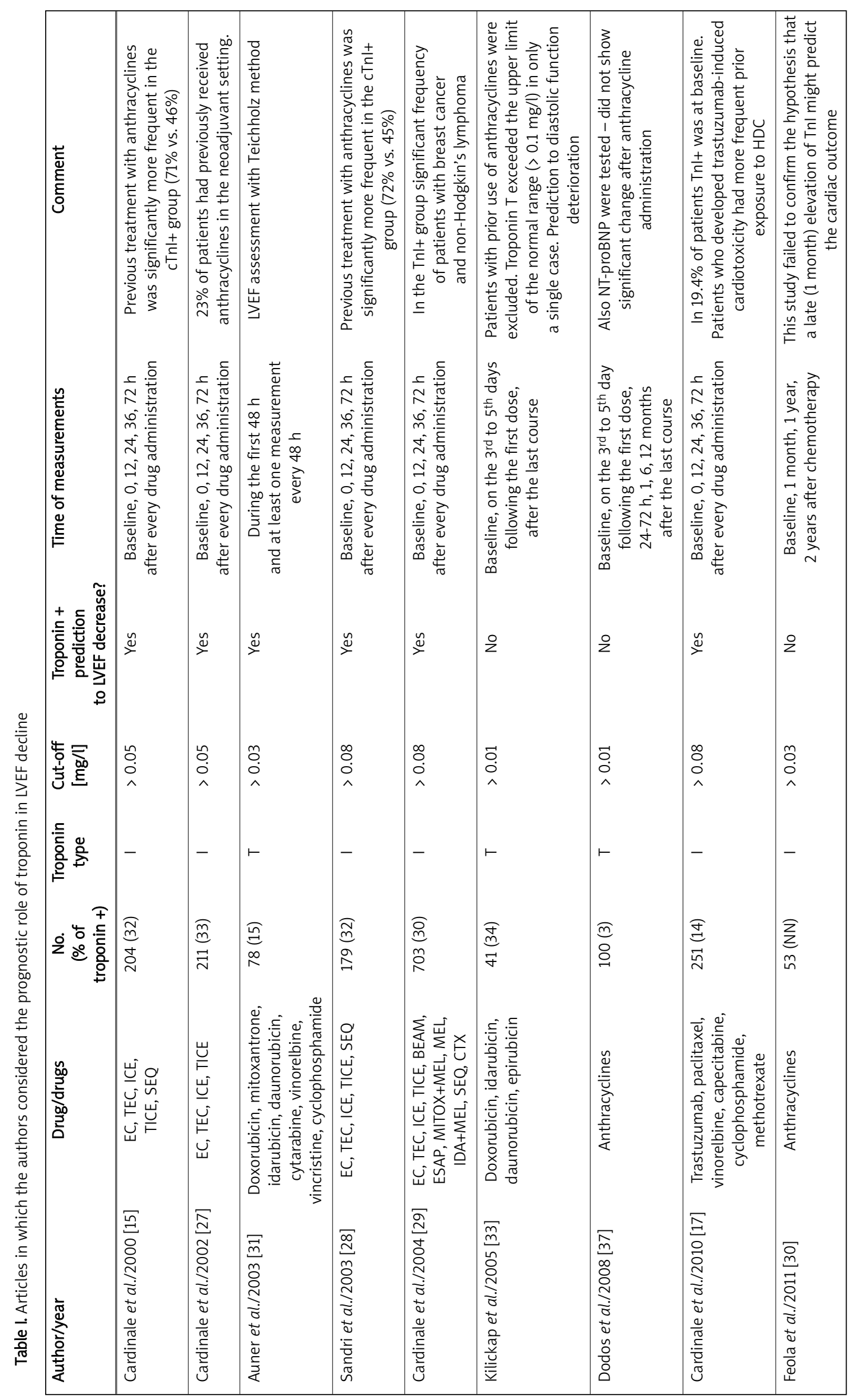




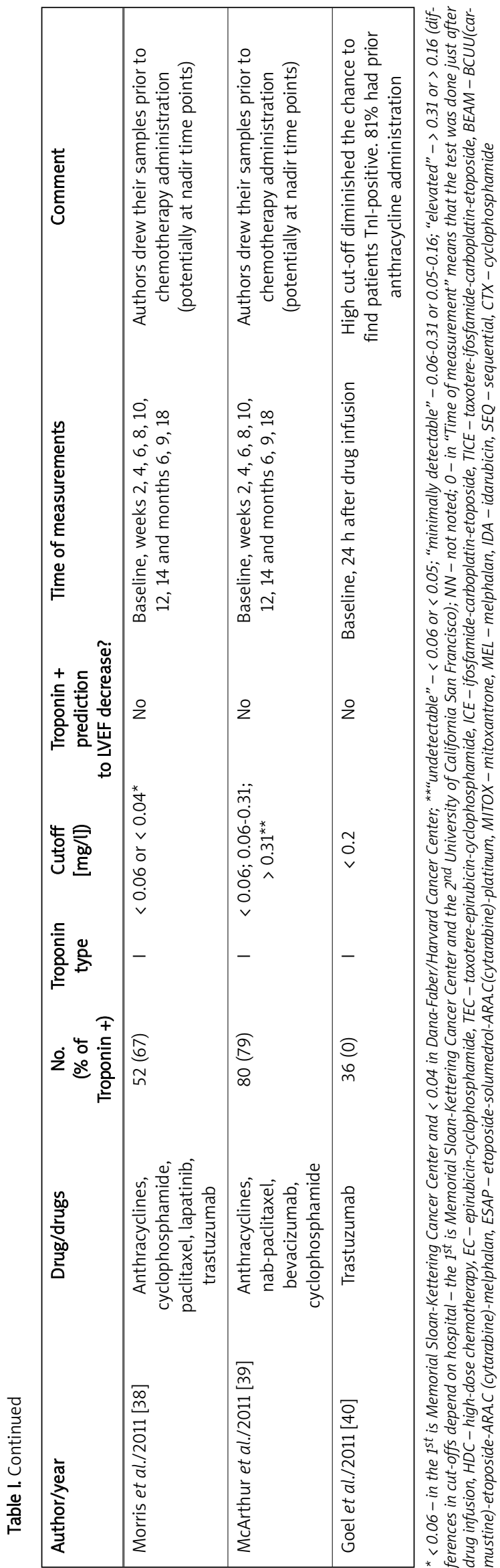

In contrast to the above studies, Kouloubinis et al. reported that levels of proANP and NT-proBNP excessively increase after chemotherapy in patients who develop heart failure during the follow-up [60].

In one of the latest studies Fallah-Rad et al. found that both troponin T and NT-proBNP did not change over time (12 months follow-up) during treatment with trastuzumab. In this study, the baseline of NT-proBNP was quite low $(27.5 \pm 2.4 \mathrm{pmol} / \mathrm{l})$, which could have been the reason for such results [61]. As other researchers proved, pretreatment plasma NT-proBNP levels were significantly higher in patients who developed severe heart failure $(534 \pm 236 \mathrm{ng} / \mathrm{l}$ vs. $105 \pm 79 \mathrm{ng} / \mathrm{l})$ [61]. The findings of this study suggest that NT-proBNP levels may play a role in early prediction of cardiotoxicity, especially in patients with a higher plasma level of this marker at the baseline [62]. Similarly, Feola et al. observed a significant LVEF reduction in patients with higher baseline plasma BNP [38].

In all of these studies the cut-offs were different. It is still quite difficult to find a proper cut-off, especially when it is commonly known that levels of NT-proBNP change with age. So far, the usage of BNP has been described in National Cancer Institute Common Terminology Criteria and Adverse Events (CTCAE), in which an elevated level of BNP suggests mild heart failure or can indicate the beginning of the process of myocarditis during chemotherapy. In the case of an elevated level of $\mathrm{BNP}$, these criteria suggest only clinical or diagnostic observation [63]. Comparisons of all studies on the use of natriuretic peptides as early biomarkers in LVEF deterioration in cancer treatment are shown in Table II.

\section{Conclusions}

Since 1997 several studies have been performed on the role of troponin in cancer treatment. Some of them have confirmed the predictive role of troponin as an early marker of further ejection fraction deterioration [16, 22, 34-36]. All of them were performed in the same center and no multicenter or international study has confirmed that hypothesis so far. In all these studies troponin was measured very frequently. In none of the other studies did the authors schedule such frequent troponin assessments [38, 46-49]. In two research studies $[46,47]$, the authors assessed troponin two weeks after drug administration, which could diminish their chance of finding troponin-positive patients.

In almost all of the studies in which the predictive role of troponin was shown, the cTnl-positive patients were in groups that had received previous anthracycline therapy [20, 22, 34, 35], apart from one [36] in which the patients who had received prior AC treatment and developed cardiotoxicity were excluded by protocol from further chemother- 


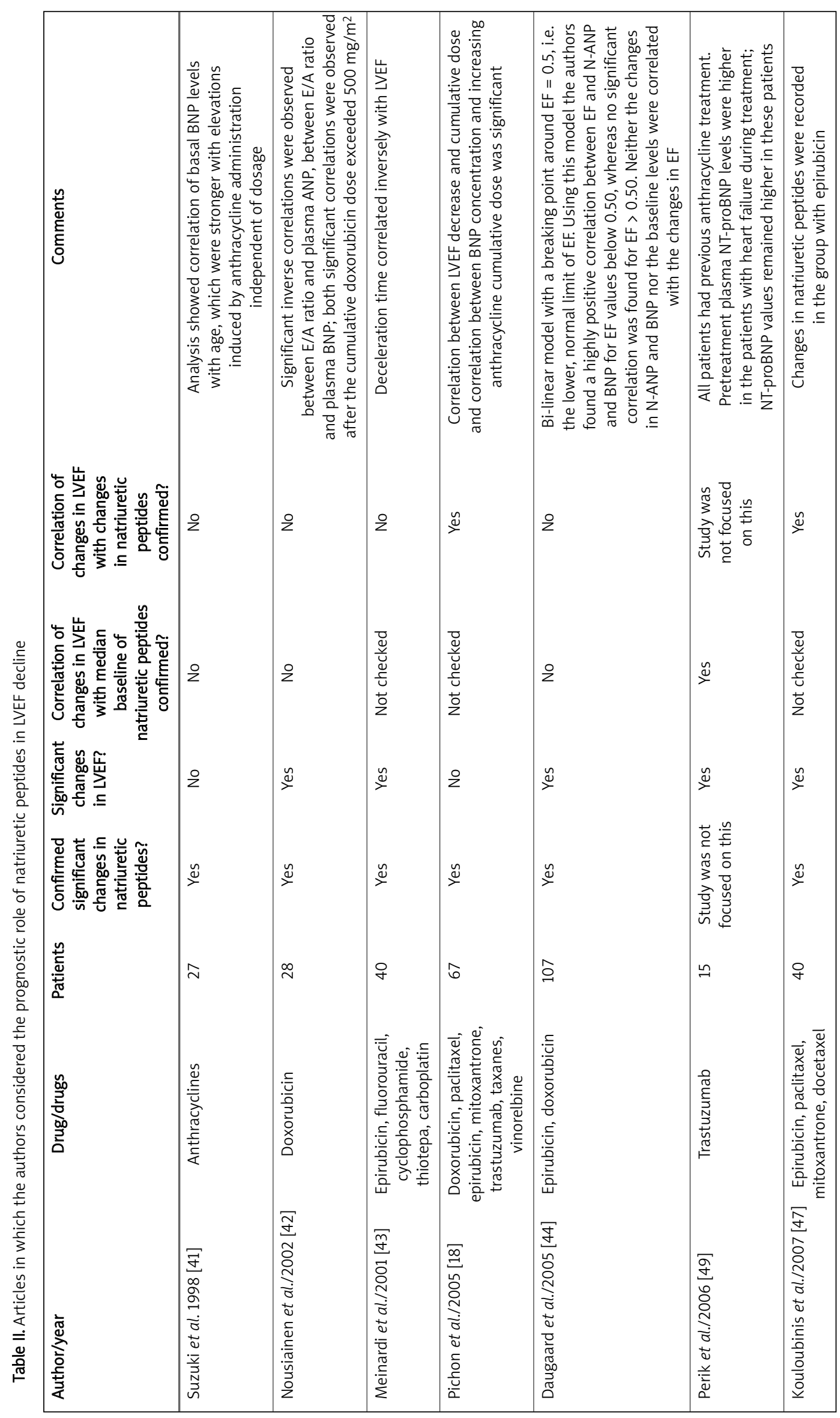




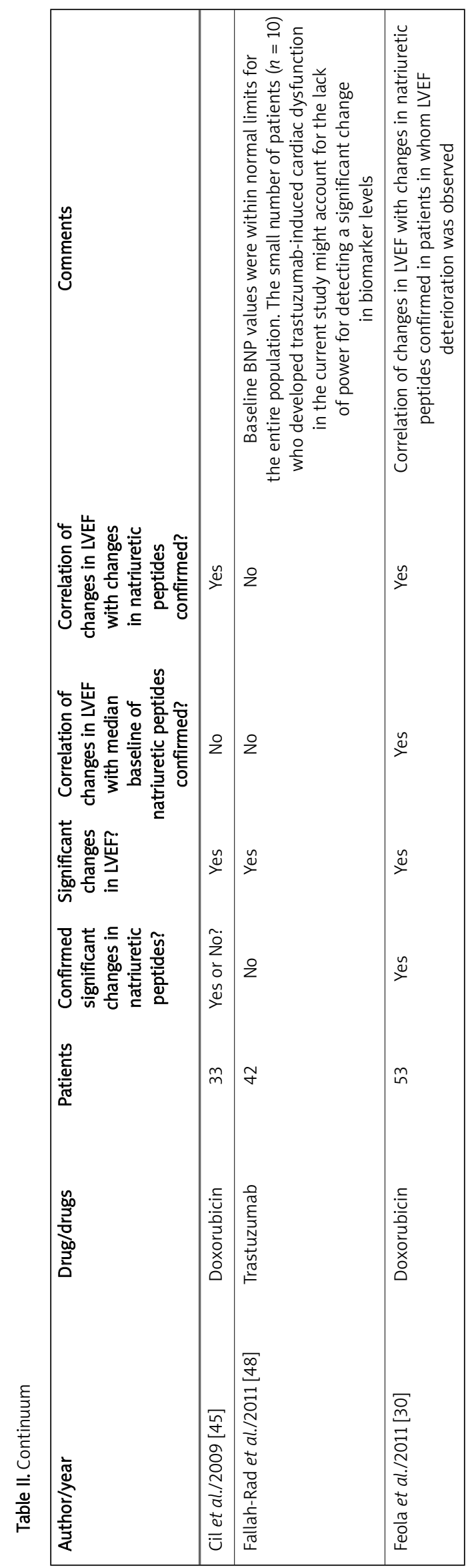

apy. Therefore patients having a greater propensity to AC-induced cardiotoxicity may have been excluded by the preliminary selection [36].

Thus, while early identification of patients at risk for acute anthracycline-related cardiac toxicity through the use of troponin holds promise for identification of patients who might benefit from preventive strategies, there is insufficient available evidence to support this approach. There are no clinical settings in which serial monitoring of serum troponin levels could be considered as a standard approach for patients receiving potentially cardiotoxic therapy, although in CTCAE both troponin T and $I$ are mentioned as markers which can suggest a mild adverse effect of oncological therapy or myocardial infarction, when the level for $\mathrm{MI}$ was exceeded [63].

A few studies of the predictive role of natriuretic peptides have shown that higher baseline concentrations of NT-proBNP can predict the development of overt heart failure after cardiotoxic chemotherapy. Many of the authors did not prove the predictive role of natriuretic peptides at all, but only showed that levels of natriuretic peptides increase during treatment, which could be explained as having been secreted by the heart in response to strain.

The conclusion drawn from data collected in studies described in this article is that regardless of the many studies about the predictive roles of troponin and natriuretic peptides, there are still many unknowns. The time point at which cardiac markers should be measured cannot be defined. This is a limitation for using the markers in clinical practice. The need to frequently obtain blood samples for troponin testing could make this approach unsuitable for patients discharged after only oneday stay. This problem seems particularly important since other studies have shown that outpatient management of high-dose chemotherapy can be safe and acceptable for the vast majority of patients and that intensive outpatient care is becoming the primary mode of care for those patients [64-67].

Apart from the foregoing CTCAE, no guidelines have been developed specifically for the definition, detection, or therapy of cardiotoxicity resulting from antineoplastic therapy. It seems that troponin I can be an interesting biochemical marker for cardiotoxic chemotherapy, especially in groups of patients who have previously had anthracycline administration or in whom a cumulative dose $\geq 300 \mathrm{mg} / \mathrm{m}^{2}$ of doxorubicin or other equivalent anthracycline was scheduled [39]. In studies where troponin level predicts further LVEF decline, cumulative doses of drugs were at least at this level [20, 34, 35, 36]. Clinicians should draw attention to patients with higher NTproBNP/BNP before chemotherapy. Cut-offs of natriuretic peptides that could play a predictive role are 
still elusive. But according to the foregoing studies, a level of NT-proBNP between $300 \mathrm{ng} / \mathrm{l}$ and $500 \mathrm{ng} / \mathrm{l}$ in serum can indicate patients with a higher propensity for further heart failure [68]. Thus both cumulative dose and higher baseline of NT-proBNP can help target a group of patients in whom cardiac monitoring should be more frequent.

Effectiveness of using biomarkers to detect and identify cardiotoxicity is still a study area. There are several multicenter trials that are ongoing concerning that subject [69]. Maybe a new hope could be high-sensitivity troponin T (hsTnT), which can be a very early predictor of cardiac damage [70, 71]. Early diagnosis of patients with higher cardiac risk is important for both oncologists and cardiologists. Of course the presence of predictive markers does not mandate cessation of a potentially lifesaving anticancer therapy. Rather, this marker may help target patients who could benefit from closer cardiac monitoring and earlier initiation of cardioprotective medical therapy.

\section{References}

1. Lipshultz SE, Colan SD, Gelber RD, Perez-Atayde AR, SalIan SE, Sanders SP. Late cardiac effects of doxorubicin therapy for acute lymphoblastic leukemia in childhood. N Engl J Med 1991; 324: 808-15.

2. Glück S. Adjuvant chemotherapy for early breast cancer: optimal use of epirubicin. Oncologist 2005; 10: 780-91.

3. Schimmel KJ, Richel DJ, van den Brink RB, Guchelaar HJ. Cardiotoxicity of cytotoxic drugs. Cancer Treat Rev 2004; 30: 181-91.

4. Gennari A, Salvadori B, Donati S, et al. Cardiotoxicity of epirubicin/paclitaxel-containing regimens: role of cardiac risk factors. J Clin Oncol 1999; 17: 3596-602.

5. Lieutaud T, Brain E, Golgran-Toledano D, et al. 5-Fluorouracil cardiotoxicity: a unique mechanism for ischaemic cardiopathy and cardiac failure? Eur J Cancer 1996; 32A: 368-9.

6. Cwikiel M, Persson SU, Larsson H, Albertsson M, Eskilsson J. Changes of blood viscosity in patients treated with 5-fluorouracil: a link to cardiotoxicity? Acta Oncol 1995; 34: 83-5.

7. Piotrowski G, Gawor R, Stasiak A, et al. Cardiac complications associated with trastuzumab in the setting of adjuvant chemotherapy for breast cancer overexpressing human epidermal growth factor receptor type 2 a prospective study. Arch Med Sci 2012; 8: 227-35.

8. Lenaz L, Page JA. Cardiotoxicity of adriamycin and related anthracyclines. Cancer Treat Rev 1976; 3: 111-20.

9. Freter CE, Lee TC, Billingham ME, Chak L, Bristow MR. Doxorubicin cardiac toxicity manifesting seven years after treatment. Case report and review. Am J Med 1986; 80: 483-5.

10. Steinherz LJ, Steinherz PG, Tan CT, Heller G, Murphy ML. Cardiac toxicity 4 to 20 years after completing anthracycline therapy. JAMA 1991; 266: 1672-7.

11. Felker GM, Thompson RE, Hare JM, et al. Underlying causes and long-term survival in patients with initially unexplained cardiomyopathy. NEJM 2000; 342: 1077-84.

12. Myers C. The role of iron in doxorubicin-induced cardiomyopathy. Semin Oncol 1998; 25: 10-4.
13. Ascensão A, Oliveira PJ, Magalhães J. Exercise as a beneficial adjunct therapy during doxorubicin treatment: role of mitochondria in cardioprotection. Int J Cardiol 2012; 156: 4-10.

14. Bien S, Riad A, Ritter CA, et al. The endothelin receptor blocker bosentan inhibits doxorubicin-induced cardiomyopathy. Cancer Res 2007; 67: 10428-35.

15. Lotrionte $M$, Palazzoni G, Natali R, et al. Appraising cardiotoxicity associated with liposomal doxorubicin by means of tissue Doppler echocardiography end-points: rationale and design of the LITE (Liposomal doxorubicinInvestigational chemotherapy-Tissue Doppler imaging Evaluation) randomized pilot study. Int J Cardiol 2009; 135: 72-7.

16. Lipshultz SE, Rifai N, Dalton VM, et al. The effect of dexrazoxane on myocardial injury in doxorubicin-treated children with acute lymphoblastic leukemia. N Engl J Med 2004; 351: 145-53.

17. Van Dalen EC, Caron HN, Dickinson HO, et al. Cardioprotective interventions for cancer patients receiving anthracyclines. Cochrane Database Syst Rev 2005; 1: CD003917.

18. Swain SM, Whaley FS, Gerber MC, et al. Cardioprotection with dexrazoxane for doxorubicin-containing therapy in advanced breast cancer. J Clin Oncol 1997; 15: 1318-32.

19. Hensley ML, Hagerty KL, Kewalramani T, et al. American Society of Clinical Oncology 2008 clinical practice guideline update: use of chemotherapy and radiation therapy protectants. J Clin Oncol 2009; 27: 127-45.

20. Cardinale D, Sandri MT, Martinoni A, et al. Left ventricular dysfunction predicted by early troponin I release after high-dose chemotherapy. J Am Coll Cardiol 2000; 36: 517-22.

21. Lipshultz SE, Rifai N, Sallan SE, et al. Predictive value of cardiac troponin $\mathrm{T}$ in pediatric patients at risk for myocardial injury. Circulation 1997; 96: 2641-8.

22. Cardinale D, Colombo A, Torrisi R, et al. Trastuzumabinduced cardiotoxicity: clinical and prognostic implications of troponin I evaluation. J Clin Oncol 2010; 28: 3910-6.

23. Pichon MF, Cvitkovic F, Hacene K, et al. Drug-induced cardiotoxicity studied by longitudinal B-type natriuretic peptide assays and radionuclide ventriculography. In Vivo 2005; 19: 567-76.

24. Verma S, Ewer MS. Is cardiotoxicity being adequately assessed in current trials of cytotoxic and targeted agents in breast cancer? Ann Oncol 2011; 22: 1011-8.

25. Roth HJ, Leithäuser RM, Doppelmayr H, et al. Cardiospecificity of the 3rd generation cardiac troponin T assay during and after a $216 \mathrm{~km}$ ultra-endurance marathon run in Death Valley. Clinl Res Cardiol 2007; 96: 359-64.

26. Kurz K, Voelker R, Zdunek D, et al. Effect of stress-induced reversible ischemia on serum concentrations of ischemiamodified albumin, natriuretic peptides and placental growth factor. Clinl Res Cardiol 2007; 96: 152-9.

27. Scharhag J, Herrmann M, Weissinger M, Herrmann W, Kindermann W. N-terminal B-type natriuretic peptide concentrations are similarly increased by 30 minutes of moderate and brisk walking in patients with coronary artery disease. Clin Res Cardiol 2007; 96: 218-26.

28. Weber M, Kleine C, Keil E, et al. Release pattern of $\mathrm{N}$-terminal pro B-type natriuretic peptide (NT-proBNP) in acute coronary syndromes. Clin Res Cardiol 2006; 95: 270-80.

29. Yu CM, Sanderson JE, Shum IO, et al. Diastolic dysfunction and natriuretic peptides in systolic heart failure. Higher ANP and BNP levels are associated with the restrictive filling pattern. Eur Heart J 1996; 17: 1694-702. 
30. Collinson PO, Moseley D, Stubbs PJ, et al. Troponin T for the differential diagnosis of ischaemic myocardial damage. Ann Clin Biochem 1993; 30: 11-6.

31. Green GB, Li DJ, Bessman ES, et al. The prognostic significance of troponin I and troponin T. Acad Emerg Med 1998; 5: 758-67.

32. Herman EH, Zhang J, Lipshultz SE, et al. Correlation between serum levels of cardiac troponin- $T$ and the severity of the chronic cardiomyopathy induced by doxorubicin. J Clin Oncol 1999; 17: 2237-43.

33. Von Hoff DD, Layard MW, Basa P, et al. Risk factors for doxorubicin-induced congestive heart failure. Ann Intern Med 1979; 91: 710-7.

34. Cardinale D, Sandri MT, Martinoni A, et al. Myocardial injury revealed by plasma troponin I in breast cancer treated with high-dose chemotherapy. Ann Oncol 2002; 13: 710-5.

35. Sandri MT, Cardinale D, Zorzino L, et al. Minor increases in plasma troponin I predict decreased left ventricular ejection fraction after high-dose chemotherapy. Clin Chem 2003; 49: 248-52.

36. Cardinale D, Sandri MT, Colombo A, et al. Prognostic value of troponin I in cardiac risk stratification of cancer patients undergoing high-dose chemotherapy. Circulation 2004; 109: 2749-54.

37. Swain SM, Whaley FS, Ewer MS. Congestive heart failure in patients treated with doxorubicin: a retrospective analysis of three trials. Cancer 2003; 97: 2869-79.

38. Feola M, Garrone O, Occelli M, et al. Cardiotoxicity after anthracycline chemotherapy in breast carcinoma: effects on left ventricular ejection fraction, troponin I and brain natriuretic peptide. Int J Cardiol 2011; 148: 194-8.

39. Keefe DL. Anthracycline-induced cardiomyopathy. Semin Oncol 2001; 28: 2-7.

40. Auner HW, Tinchon C, Linkesch W, et al. Prolonged monitoring of troponin $\mathrm{T}$ for the detection of anthracycline cardiotoxicity in adults with hematological malignancies. Ann Hematol 2003; 82: 218-22.

41. Lang RM, Bierig M, Devereux RB, et al. Recommendations for chamber quantification. Eur J Echocardiogr 2006; 7 79-108.

42. Kilickap S, Barista I, Akgul E, et al. cTnT can be a useful marker for early detection of anthracycline cardiotoxicity. Ann Oncol 2005; 16: 798-804

43. Fouad FM, Slominski JM, Tarazi RC. Left ventricular diastolic function in hypertension: relation to left ventricular mass and systolic function. J Am Coll Cardiol 1984; 3: 1500-6.

44. Inouye I, Massie B, Loge D, et al. Abnormal left ventricular filling: an early finding in mild to moderate systemic hypertension. Am J Cardiol 1984; 53: 120-6.

45. Raev DC. Which left ventricular function is impaired earlier in the evolution of diabetic cardiomyopathy? An echocardiographic study of young type I diabetic patients. Diabetes Care 1994; 17: 633-9.

46. Dodos F, Halbsguth T, Erdmann E, Hoppe UC. Usefulness of myocardial performance index and biochemical markers for early detection of anthracycline-induced cardiotoxicity in adults. Clin Res Cardiol 2008; 97: 318-26.

47. Morris PG, Chen C, Steingart R, et al. Troponin I and $C$-reactive protein are commonly detected in patients with breast cancer treated with dose-dense chemotherapy incorporating trastuzumab and lapatinib. Clin Cancer Res 2011; 17: 3490-9.

48. McArthur HL, Rugo H, Nulsen B, et al. A feasibility study of bevacizumab plus dose-dense doxorubicin-cyclophosphamide (AC) followed by nanoparticle albumin- bound paclitaxel in early-stage breast cancer. Clin Cancer Res 2011; 17: 3398-407.

49. Goel S, Simes RJ, Beith JM. Exploratory analysis of cardiac biomarkers in women with normal cardiac function receiving trastuzumab for breast cancer. Asia Pac J Clin Oncol 2011; 7: 276-80.

50. Mukoyama M, Nakao K, Hosoda K, et al. Brain natriuretic peptide as a novel cardiac hormone in humans. J Clin Invest 1991; 87: 1402-12.

51. Nishikimi T, Kuwahara K, Nakao K. Current biochemistry, molecular biology, and clinical relevance of natriuretic peptides. J Cardiol 2011; 57: 131-40.

52. Levin ER, Gardner DG, Samson WK. Natriuretic peptides. N Engl J Med 1998; 339: 321-8.

53. Feola M, Lombardo E, Testa M, et al. Prognostic factors of mid-term clinical outcome in congestive heart failure patients discharged after acute decompensation. Arch Med Sci 2012; 8: 462-70.

54. Suzuki T, Hayashi D, Yamazaki T, et al. Elevated B-type natriuretic peptide levels after anthracycline administration. Am Heart J 1998; 136: 362-3.

55. Nousiainen T, Vanninen E, Jantunen E, et al. Natriuretic peptides during the development of doxorubicin-induced left ventricular diastolic dysfunction. J Intern Med 2002; 251: 228-34.

56. Meinardi MT, van Veldhuisen DJ, Gietema JA, et al. Prospective evaluation of early cardiac damage induced by epirubicin-containing adjuvant chemotherapy and locoregional radiotherapy in breast cancer patients. J Clin Oncol 2001; 19: 2746-53.

57. Daugaard G, Lassen U, Bie P, et al. Natriuretic peptides in the monitoring of anthracycline induced reduction in left ventricular ejection fraction. Eur J Heart Fail 2005; 7: 87-93.

58. Cil T, Kaplan AM, Altintas A, Akin AM, Alan S, Isikdogan A. Use of $\mathrm{N}$-terminal pro-brain natriuretic peptide to assess left ventricular function after adjuvant doxorubicin therapy in early breast cancer patients: a prospective series. Clin Drug Investig 2009; 29: 131-7.

59. Tanindi A, Demirci U, Tacoy G, et al. Assessment of right ventricular functions during cancer chemotherapy. Eur J Echocardiogr 2011; 12: 834-40.

60. Kouloubinis A, Kaklamanis L, Ziras N, et al. ProANP and NT-proBNP levels to prospectively assess cardiac function in breast cancer patients treated with cardiotoxic chemotherapy. Int J Cardiol 2007; 122: 195-201.

61. Fallah-Rad N, Walker JR, Wassef A, et al. The utility of cardiac biomarkers, tissue velocity and strain imaging, and cardiac magnetic resonance imaging in predicting early left ventricular dysfunction in patients with human epidermal growth factor receptor II-positive breast cancer treated with adjuvant trastuzumab therapy. J Am Coll Cardiol 2011; 57: 2263-70.

62. Perik PJ, Lub-De Hooge MN, Gietema JA, et al. Indium-111labeled trastuzumabscintigraphy in patients with human epidermal growth factor receptor 2-positive metastatic breast cancer. J Clin Oncol 2006; 24: 2276-82.

63. National Cancer Institute. Common Terminology Criteria for Adverse Events v.3.0and v.4.03 (CTCAE). Available at:http://evs.nci.nih.gov/ftp1/CTCAE/CTCAE_4.03_201006-14_QuickReference_5x7.pdf.

64. Dix SP, Geller RB. High-dose chemotherapy with autologous stem cell rescue in the outpatient setting. Oncology (Williston Park) 2000; 14: 171-85.

65. Meisenberg BR, Miller WE, McMillan R, et al. Outpatient high-dose chemotherapy with autologous stem-cell rescue for hematologic and nonhematologic malignancies. J Clin Oncol 1997; 15: 11-7. 
66. Dollinger M. Guidelines for hospitalization for chemotherapy. Oncologist 1996; 1: 107-11.

67. Savoie ML, Nevil TJ, Song KW, et al. Shifting to outpatient management of acute myeloid leukemia: a prospective experience. Ann Oncol 2006; 17: 763-8.

68. Newby LK, Rodriguez I, Finkle J, et al. Troponin measurements during drug development-considerations for monitoring and management of potential cardiotoxicity: an educational collaboration among the Cardiac Safety Research Consortium, the Duke Clinical Research Institute, and the US Food and Drug Administration. Am Heart J 2011; 162: 64-73.

69. A service of the U.S. National Institutes of Health. www.clinicaltrials.gov

70. deFilippi CR, de Lemos JA, Christenson RH, et al. Association of serial measures of cardiac troponin $T$ using a sensitive assay with incident heart failure and cardiovascular mortality in older adults. JAMA 2010; 304: 2494-502.

71. Palazzuoli A, Gallotta M, Quatrini I, et al. Natriuretic peptides (BNP and NT-proBNP): measurement and relevance in heart failure. Vasc Health Risk Manag 2010; 6: 411-8. 